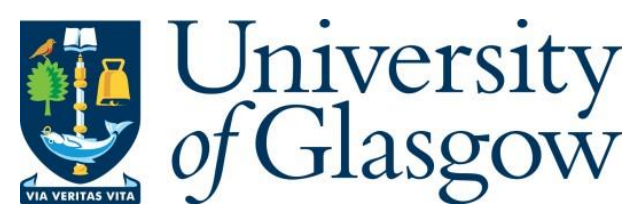

Krämer, S., and Roski, S. (2017) Difference-making grounds. Philosophical Studies, 174(5), pp. 1191-1215.

There may be differences between this version and the published version. You are advised to consult the publisher's version if you wish to cite from it.

http://eprints.gla.ac.uk/140558/

Deposited on: 4 May 2017

Enlighten - Research publications by members of the University of Glasgow http://eprints.gla.ac.uk 


\title{
DIFFERENCE-MAKING GROUNDS
}

\author{
STEPHAN KRÄMER AND STEFAN ROSKI
}

This is the penultimate version of an article accepted for publication by Philosophical Studies. Please quote only from the published version.

The final publication is available at http://link. springer.com

\begin{abstract}
We define a notion of difference-making for partial grounds of a fact in rough analogy to existing notions of difference-making for causes of an event. Using orthodox assumptions about ground, we show that it induces a non-trivial division with examples of partial grounds on both sides. We then demonstrate the theoretical fruitfulness of the notion by applying it to the analysis of a certain kind of putative counter-example to the transitivity of ground recently described by Jonathan Schaffer. First, we show that our conceptual apparatus of difference-making enables us to give a much clearer description than Schaffer does of what makes the relevant instances of transitivity appear problematic. Second, we suggest that difference-making is best seen as a mark of good grounding-based explanations rather than a necessary condition on grounding, and argue that this enables us to deal with the counter-example in a satisfactory way. Along the way, we show that Schaffer's own proposal for salvaging a form of transitivity by moving to a contrastive conception of ground is unsuccessful. We conclude by sketching some natural strategies for extending our proposal to a more comprehensive account of grounding-based explanations.
\end{abstract}

\section{INTRODUCTION}

There is a familiar idea that grounding is in certain respects analogous to causation. In particular, it has been suggested that grounding and causation are alike in that they are both explanation-backing relations.1 For causation, the idea would be roughly as follows. Sometimes, an event $c$ causes another $e$. In at least some of these cases, it is correct to say that $e$ occurred because $c$ occurred. Moreover, the truth of this becauseclaim is owed at least in part to the causal relationship between $c$ and $e$. And at least in

1 A picture roughly like that to be described is proposed by Schaffer (2016) and Audi (2012a b). See also Schnieder (2015, 2010). 
some cases, a true because-claim whose truth is thus owed to an instance of causation may be used to give an adequate causal explanation of why the event $e$ occurred. ? $^{2}$

For grounding, a parallel idea might run as follows. Sometimes, a fact $f$ grounds a fact $g$. In at least some of these cases, it is correct to say that $g$ obtains because $f$ obtains. Moreover, the truth of this because-claim is owed at least in part to the grounding relationship between $f$ and $g$. And at least in some cases, a true becauseclaim whose truth is thus owed to an instance of grounding may be used to give an adequate grounding explanation of why the fact $g$ obtains.

We may say that a cause of an event $e$ is causal-explanatorily relevant to $e$ iff it underlies an adequate causal explanation in the way described. And we may say that a ground of a fact $g$ is grounding-explanatorily relevant to $g$ iff it underlies an adequate grounding explanation in the way described.

It is sometimes suggested, with respect to causal explanation, that only a certain elite subclass of the causes of a given event are causal-explanatorily relevant, and that the members of this subclass may be singled out by a criterion of difference-making. Not every causal influence on a given event, however minor and remote, can play a role in explaining why the event occurred, but only those that make a difference to its occurrence. This idea is central in particular to Michael Strevens' much-discussed theory of causal explanation. $]^{3}$ A somewhat less controversial, if also less informative version of the claim is that there is a significant distinction between two kinds of causes, naturally described in terms of difference-making, which has some important role to play in the theory of causation and causal explanation. It might be held, for example, that although non-difference-making causes are capable of figuring in explanations per $s e$, they will generally not figure in good explanations. $4^{4}$ On this kind of view, the notion of difference-making still seems to be of significant theoretical interest in the study of causal explanation.

Given the analogy between grounding and causation, a number of questions suggest themselves. Is there a natural way to explicate the notion of difference-making in the application to grounding? If so, is the resulting notion theoretically fruitful? In particular, is it plausible to suppose that it relates to grounding explanations in something

2 In the debate about causal explanation, a view like this is endorsed, for example, by Strevens (2008), Woodward (2003) and Ruben (2012).

3 See Strevens (2008), in particular ch. 2. Criteria of difference-making play an important role in the debate on causation in general-sometimes not quite with the same role they have in Strevens; cf. e.g. Lewis (1973. 160f), and List and Menzies (2009). We will briefly come back to this in sec. 7 below.

4 This is one way to understand Lewis's view on the matter; cf. Lewis (1986a). 
like the way in which causal difference-making has been claimed to relate to causal explanation? We argue in this paper that the answer to all three questions is yes.

We begin by introducing a natural abstract characterization of a notion of difference-making for grounds in rough analogy to Strevens' work on causal differencemaking (sec. 2). Next, we establish some basic observations about that notion. Using orthodox assumptions about ground, we show that there are instances of differencemaking ground as well as instances of non-difference-making ground, and that difference-making partial grounding is not transitive (sec. 3). The structure of one of the examples we use to show this is strongly reminiscent of some of the putative counterexamples to the transitivity of grounding described by Schaffer (2012). We show how our conceptual apparatus of difference-making enables us to give a much clearer description than Schaffer can offer of what makes the relevant instances of transitivity appear problematic, thereby establishing a first, significant theoretical payoff of the notion (sec. 4). We then turn to the relation between difference-making and explanation, and suggest that by taking our notion of difference-making to be a mark of good grounding explanations rather than a necessary condition on grounding, the apparent counterexamples to the transitivity of ground can be dealt with in a satisfactory way (sec. 5). This marks a second theoretical payoff, which is especially significant because, as we show in sec. 6. Schaffer's own proposal for avoiding the counter-examples by moving to a contrastive conception of ground is unsuccessful. Finally, we sketch some natural avenues for further developments of our account, for instance to accommodate intuitions about the proportionality of explanantia with respect to their explananda (sec. 7).

\section{A Notion of Difference-Making For Ground}

We begin by making explicit some basic assumptions about grounding that will be in place throughout the paper and that represent orthodoxy in the current debate. 5 We take grounding to be a kind of non-causal priority that is conveyed by certain uses of 'because', 'in virtue of', and cognate phrases that are widespread in philosophical discourse. Standard examples include the claim that a given object is coloured because it is red, or that a given object is red or round because it is round. We shall assume that grounding is a relation obtaining among facts..$^{6}$ We can then distinguish between

5 For reasons of space, we have to assume a basic familiarity with the notion of grounding. For general introductory overviews see, e.g., Correia and Schnieder (2012) as well as Trogdon (2013).

6 Some authors prefer to think of ground as expressed by a sentential connective, and deny that there is, strictly speaking, a relation of grounding obtaining between facts. We make this assumption purely for ease of expression; our arguments as well as the idea of grounding as explanation-backing may be 
the relation of partial ground, which is a binary relation between some fact $f$ and another fact $g$, and that of full ground, which is a relation between some plurality of facts $f_{1}, f_{2}, \ldots$ and another fact $g$. We sometimes also write $P<Q$ to say that the fact that $P$ partially grounds the fact that $Q$, and $\Gamma<Q$ to say that the fact that $P_{1}$, the fact that $P_{2}$, ... together fully ground the fact that $Q$, where $\Gamma=\left\{\right.$ the fact that $P_{1}$, the fact that $P_{2}$, ...\}. 7 As is standard, we assume that for a fact to partially ground another is for it to be a member of some full ground of that fact.

Our task is to devise a notion of difference-making that applies to the grounds of a given fact. To see how this might be done, it seems both natural and methodologically advisable to take a cue from the philosophy of science, where the analogous question with respect to the causes of a given event has been the subject of extensive discussion. A particularly helpful suggestion is contained in recent work by Strevens (2008), who describes a general template that any account of causal difference-making instantiates:

All such accounts have a common form. To determine whether a causal influence $c$ makes a difference to an explanandum $e$, a comparison is made between two scenarios: the actual scenario, in which $c$ is present, and a nonactual scenario in which $c$ is not present. The facility with which e occurs in each scenario is evaluated. If it varies, then $c$ is classified as a difference-maker. (Strevens, 2008; 55)

Indeed, this template seems to us to be general enough to also apply, with minor modifications, in the realm of grounding. We have found the most fruitful way of instantiating Strevens' pattern to be as follows. A scenario is a collection of facts and/or mere states of affairs (the non-obtaining cousins of facts)..$^{8} \mathrm{~A}$ fact is considered present in a scenario just in case it is a member of the scenario. Although talk of the facility with which a fact $g$ obtains in a scenario sounds like a gradual matter, we shall, in the first instance, interpret it in terms of the binary distinction of whether some part of the scenario is a full ground of $g$. For the time being, the parts of a scenario may here simply be identified with its subsets; depending on subtle details in the theory of ground, there may be reasons also to allow other kinds of scenarios as parts of a scenario.9

easily transposed to the alternative setting. For discussion of the matter, see (Correia and Schnieder, 2012: 10ff).

7 The notation is taken over from Fine (2012b); note, though, that Fine prefers not to think of ground as a relation between facts and hence does not use the symbolism to abbreviate fact-talk.

8 Although we prefer to allow for scenarios including mere states of affairs, this is not necessary for our purposes, so readers with ontological qualms about such entities need not be concerned.

9 The matter is taken up in the appendix. 
To determine whether a ground $f$ makes a difference to a groundee $g$, we now compare scenarios in which $f$ is present with ones in which $f$ is not present. A natural idea is to consider any actual scenario $S$ in which $f$ is present, and compare it with the result $S^{\prime}=S \backslash\{f\}$ of removing $f$ from $S . .^{10}$ We then compare the facility with which $g$ obtains in both $S$ and $S^{\prime}$. That is, we ask whether $g$ is fully grounded by a subset of $S$, and whether $g$ is fully grounded by a subset of $S^{\prime}$. Since every subset of $S^{\prime}$ is a subset of $S$, either the answer is the same with respect to both $S$ and $S^{\prime}$, or the answer is positive with respect to $S$ and negative with respect to $S^{\prime}$. In the latter case, the facility with which $g$ obtains varies between $S$ and $S^{\prime}$, and we conclude that $f$ is a difference-making ground of $g$. On the other hand, if such variation does not occur for any scenario $S$, we conclude that $f$ is a non-difference-making ground of $g$. We therefore propose the following definition of difference-making partial grounds: $: 11$

(Df. $\prec_{D}$ ): The fact that $P$ is a difference-making partial ground of the fact that $Q$ $\left(P \prec_{D} Q\right)=_{\text {df. }}$

for some scenario $S$ which contains a full ground of $Q, S \backslash$ the fact that $P$ \} does not contain a full ground of $Q$.

It seems to us that a partial ground that does not satisfy our definition of differencemaking could indeed quite naturally be described as not making a difference. ${ }^{12}$ For

$\overline{10}$ We are then not strictly speaking comparing an actual scenario with a non-actual one, as Strevens would have us do. We could do so, however, by replacing $f$ with some non-obtaining state of affairs. But since this produces exactly the same results as our simpler procedure, we stick to the latter. Note also that Strevens speaks of the actual scenario, whereas we have many, namely any collection of facts. We see no attractive way of amending our procedure to appeal to just one actual scenario. Finally, many people's first idea for cashing out talk of comparison of actual scenarios and non-actual versions of them will be in terms of possible worlds and counterfactuals. But this is by no means mandatory; indeed, Strevens' own account also does not explicate his talk of comparison of actual and nonactual scenarios in terms of counterfactuals and possible worlds (cf. Strevens (2008: 111ff)). For our purposes, the present way of cashing Strevens' idea out is much more fruitful. We shall have some use for counterfactuals in considering alternative possibilities later on, though (cf. sec. 4 ).

11 There is an obvious strengthening of this notion of a difference-making partial ground, on which it is required that for all full grounds that include the fact that $P$, removing that fact yields a collection that does not contain a full ground anymore. However, given the common assumption that if $\Gamma<P$ and $\Delta<P$, then $\Gamma \cup \Delta<P$, this would imply that any fact which has several full grounds-which, at least assuming transitivity, is true of the vast majority of facts-has no difference-making partial grounds. So the resulting notion of difference-making would not be very useful.

12 Note that (Df. $\prec_{D}$ ) has the consequence that any difference-making partial ground is a partial ground, as one would have hoped. For suppose $S$ contains a full ground of $Q$, but $S \backslash\{$ the fact that $P$ \} does not. Let $\Gamma \subseteq S$ be such that $\Gamma<Q$. Then $\Gamma$ is not a subset of $S \backslash\{$ the fact that $P\}$. It follows that the fact that $P$ is a member of $\Gamma$, and hence that $P \prec Q$. 
suppose $P<Q$, but $P k_{D} Q$. Then for any full ground $\Gamma$ of $Q$ that includes the fact that $P$, removing that fact from $\Gamma$ results in a scenario still containing a full ground of $Q$. In this way, the fact that $P$ is seen to be at best an idle wheel in any scenario fully grounding $Q$. Another way to make the point is by asking what we can add to the fact that $P$ so as to obtain a full ground of the fact $Q$. Given that $P k_{D} Q$, any collection of facts that will do the job already contains a full ground of $Q$. So the fact that $P$ really does not get us any closer to the fact that $Q 4^{13}$

Note that for all we have said so far, there may not actually be any instances of nondifference-making partial ground. And indeed, given what we have just said about what such putative grounds would be like, one may have the intuition that these features would precisely disqualify the relevant facts as candidate grounds. As we shall see in the next section, however, given only fairly orthodox, if not uncontroversial assumptions about grounding, it can be shown that examples of both difference-making and nondifference-making partial grounding exist.

\section{Difference-Making and Transitivity}

In general, whether one should take there to be instances both of difference-making grounding and of non-difference-making grounding depends on one's preferred theory of ground. However, it would take a highly unorthodox view of ground to deny that there are instances of difference-making partial grounding. For take any instance in which a single fact that $P$ is a full ground of a fact that $Q$. Then since removing the fact that $P$ from the collection \{the fact that $P$ \} leaves an empty set, it is clear that the fact that $P$ is a difference-making partial ground of the fact that $Q 4^{14}$ For example, it is standardly supposed that a typical true disjunction $P \vee Q$ is grounded by each of its

13 In Krämer 2016), a similar notion of something getting us closer to the truth of a proposition is employed in formulating a criterion of evidential relevance. Given that the notions of relevance and difference-making seem to be very closely related, this may provides some additional motivation for our approach to difference-making, and hints at the possibility of a unified account of differencemaking and relevance.

14 Fine (2012a 47f) suggests that some facts may be zero-grounded, which is supposed to amount to being grounded by the empty set of facts, and distinguished from being ungrounded. If so, then it may be that not every case in which a single fact fully grounds another gives rise to a case of difference-making partial grounding. However, zero-grounding, if there is such a thing, is supposed to be a feature of only a rather special and rare sort of fact, so our general point is not threatened. For simplicity, we tacitly exclude the possibility of zero-grounding in what follows. 
true disjuncts ${ }^{15}$ So if it is a fact that $P$, then $P<P \vee Q$ and hence $P<P \vee Q$. But $\emptyset \nless P \vee Q$, so the fact that $P$ is a difference-making partial ground of the fact that $P \vee Q$. For a second example, take any true conjunction $P \wedge Q$ where the fact that $P$ and the fact that $Q$ are distinct and ground-theoretically independent facts: neither helps ground the other in any way. Then on any standard view of the logic of ground, $P<P \wedge Q$ because $P, Q<P \wedge Q$. But $P \nless P \wedge Q$, for otherwise it follows that the fact that $P$ is, or grounds, the fact that $Q$. So the fact that $P$ is a difference-making partial ground of the fact that $P \wedge Q$.

It would be easier to deny that there are instances of non-difference-making partial ground. Nevertheless, there are quite strong reasons for thinking that there are such instances, and indeed that there is a systematic way of producing examples of nondifference-making partial ground. For given any two suitably ${ }^{16}$ independent facts that $P$ and that $Q$, plausible assumptions concerning the logic of ground allow us to argue that

$$
\begin{aligned}
& \text { (1): } P<Q \vee(P \wedge Q) \\
& \text { (2): } P{ }{ }_{D} Q \vee(P \wedge Q)
\end{aligned}
$$

For (1), note that as before, $P<P \wedge Q$, and by the disjunction principle, $P \wedge Q<$ $Q \vee(P \wedge Q)$. Using the following, relatively weak form of the claim that grounding is transitive, we obtain (1).

$$
\text { ( } \left.\mathbf{T}_{1}\right): \text { If } P<Q \text { and } Q<R \text {, then } P<R
$$

An informal argument for (2) may now be given as follows. Let $\Gamma \cup\{P\}<Q \vee(P \wedge Q)$. Note that $\Gamma \cup\{P\}$ must 'contain enough' to ground $Q$. Since the fact that $P$, by the assumption of ground-theoretic independence, cannot be of help in this, $\Gamma \backslash\{P\}$ must contain a scenario that fully grounds $Q$. But by the disjunction principle and the transitivity principle

$$
\left(\mathbf{T}_{2}\right): \text { If } \Gamma<Q \text { and } Q<R \text {, then } \Gamma<R
$$

it follows that this scenario will then be a full ground of $Q \vee(P \wedge Q)$. In this way, the fact that $P$ may be shown to be an idle member of any full ground of $Q \vee(P \wedge Q)$, and thus to be a non-difference-making partial ground of $Q \vee(P \wedge Q)$.

15 When we speak of standard assumptions in the (propositional) logic of ground, we mean assumptions that are explicitly endorsed in both Correia (2010) and Fine (2012a), which are so far the only reasonably developed systems for the propositional logic of ground. We give more precise versions of our informal arguments here by reference to these systems in the appendix.

16 The exact condition of independence required varies slightly with the details of the logic of ground assumed. Roughy speaking, it is sufficient to choose facts with disparate subject matters, such as that this ball is red and that that chair is brown. Details are given in the appendix. 
We would now like to describe a concrete example of non-difference-making partial ground, which is roughly analogous in structure to the kind of case just discussed, and which is moreover strongly reminiscent of a case described by Jonathan Schaffer (2012 126) ${ }^{17}$ Consider a ball $b$ which is everywhere red, except for one tiny green spot $s$, constituting less than $1 \%$ of $b$ 's surface area. Call the exact overall distribution of colours over its surface area $d$. We also appeal to a property of being largely-red, which we understand to be a disjunctive colour distribution property whose disjuncts are those partial or complete exact distributions of colours over the surface area of $b$ which render at least $99 \%$ of $b$ 's surface red. Note that $d$ is one of those disjuncts. Then

(4): $b$ is green in spot $s<b$ has colour distribution $d$.

(5): $b$ has colour distribution $d<b$ is largely-red.

(6): $b$ is green in spot $s<b$ is largely-red.

(4) is plausible since part of what it takes for $b$ to have $d$ is for $b$ to be green in spot $s$; the facts that $b$ is green in spot $s$ and that $b$ is red everywhere else jointly fully ground the fact that $b$ has colour distribution $d$. This is roughly analogous to the grounding of a conjunction by its conjuncts. (5) is plausible since one way to be largely-red is to have colour distribution $d$. This is in effect an instance of a disjunction being grounded by its true disjuncts. (6) follows from (4) and (5) by the transitivity principle $\left(\mathrm{T}_{1}\right)$.

Assuming that (6) is indeed true, it appears to be an instance of non-differencemaking partial ground. To see this, we may ask what must be added to the fact that $b$ is green in spot $s$ to obtain a full ground of the fact that $b$ is largely-red. Anything of that sort will have to ensure on its own that $99 \%$ of the surface area of $b$ is red, and thus will on its own constitute a full ground of the fact that $b$ is largely-red. (It may help to imagine you are painting the ball. If you start with painting the spot $s$ green, then the amount of red colour you have to apply after that in order to make the ball largely-red would have been sufficient to ensure that $b$ is largely-red even if you had not already painted $s$ green.)

In both of our examples, the premises to the transitivity-based inference are examples of difference-making partial ground. In the first, logical example, the premises were that $P \prec P \wedge Q$, which was already shown to be an instance of difference-making, and that $Q<Q \vee(P \wedge Q)$, which is a case of full ground, and therefore automatically of difference-making ground. In the second example, consider first premise (4). We

17 We have in mind Schaffer's case of the dented sphere. His particular example suffers from some special problems not affecting the example we describe below, which is why we prefer to focus on our case. Schaffer describes two more putative counter-examples to transitivity, which we do not discuss here. For criticism of the examples, see e.g. Litland (2013). 
observed above that the facts that $b$ is green in spot $s$ and that $b$ is red everywhere else jointly fully ground the fact that $b$ has colour distribution $d$. But clearly, the fact that $b$ is red everywhere else is not on its own a full ground of the fact that $b$ has $d$. Premise (5) is again an example of full ground. As a result, both of our examples show that on the proposed notion of difference-making, the relation of difference-making partial ground is not transitive.

It might thus be suggested that rather than taking the examples to establish the existence of non-difference-making partial ground, we should take them to establish the non-transitivity of partial ground. And indeed, Schaffer offers his analogous case as a counter-example to the transitivity of partial ground (cf. Schaffer 2012: p. 127f). And it must be admitted that especially in the second example, the because-claim corresponding to the putative non-difference-making instance of grounding sounds at best suspect:

(7): $b$ is largely-red partly because $b$ is green in spot $s$.

So it might be claimed that we have actually just rediscovered Schaffer's reasons for doubting the transitivity of ground, and perhaps identified an interesting structural feature of partial grounds, namely that they are all difference-making in our sense (and hence that (3) and (6) are not cases of grounding, after all).

We propose a way to defend the transitivity of ground, and thus the existence of nondifference-making grounds, against this objection in sec. 5 below. But before that, we want to highlight a way in which our understanding of difference-making in application to grounds is theoretically fruitful which does not depend on transitivity.

\section{Difference-Making and Subversive Double Agents}

Consider again our second potential example of a non-difference-making partial ground:

(6): $b$ is green in spot $s<b$ is largely-red

It should be conceded on all sides that this is at best quite a peculiar and somehow second-rate instance of grounding, and prior to deciding whether to count it a case of grounding, we should get as clear as possible about what makes the case so peculiar. Only then will we have a clear understanding of the reasons we may have for asserting or denying (6).

Schaffer offers a helpful account of the peculiarity of his analogue for (6) (cf. Schaffer 2012: p. 127). Transposed to our case, it reads as follows:

(i) The presence of the green spot makes no difference to the largely-redness of the ball. (ii) The ball would be largely-red either way. (iii) The presence of the green spot in no way helps support the largely-redness of the ball, but (iv) is if 
anything a threat to the largely-redness of the ball. (v) The ball is largely-red despite the green spot, not because of it.

Remark (i) is, we agree, a very natural thing to say. However, it would be good to have an explication of just how we are to understand the talk of difference-making here. Perhaps we may read Schaffer as offering (ii) as an explication of (i) ${ }^{18}$ However, it is clear that the kind of counterfactual dependence at issue in (ii) is not in general a requirement for grounding. For instance, the fact that snow is white grounds the fact that snow is white or grass is green. But it is not the case that if snow were not white, grass would not be green, and so the disjunctive fact that snow is white or grass is green would obtain either way - whether snow is white or not.

This particular failure of counterfactual dependence is akin to failures of counterfactual dependence for causation in cases of causal overdetermination. Indeed, we might say that the disjunctive fact that snow is white or grass is green is ground-theoretically overdetermined since both constituent disjuncts obtain. Since (6) and the grounding relationships by which it is mediated do not seem to involve overdetermination, one might think that they also should not give rise to a failure of counterfactual dependence

But there are many clear cases of grounding without counterfactual dependence in which no overdetermination is involved. Thus, if it is true that $P$, then $P<P \vee \neg P$. Still, it is not true that if $P$ were false, it would be false that $P \vee \neg P$. At the same time, there is clearly no overdetermination involved here. We need not even choose a logical truth as the groundee. Suppose John always comes by bus or by bike. Suppose today he came by bus, but if he had not come by bus, he would have come by bike. Then John came by bus $<$ John came by bus or by bike. But there is neither counterfactual dependence nor overdetermination involved.

It might still be, of course, that there is a range of special cases in which grounding can only plausibly be asserted provided that counterfactual dependence holds. But it is hard to see why (6) should belong to such a special subclass. For the putative grounding relationship is mediated exactly by the kind of disjunct-disjunction relationship, which

18 Although this reading is not mandatory, it seems quite natural. As we have already mentioned, it is quite common to explicate difference-making in terms of counterfactual dependence in something like this way. (For a classic statement of this intuition in the case of causal difference-making, see Lewis (1986b. 161-2).) More or less every way of understanding the counterfactual in (ii) gives quite a plausible claim, moreover. For example, it is true that if it were not the case that the ball is green in spot $s$, the ball would still be largely-red.

19 Schaffer's remark in his (2016, 31) that in the case of a disjunction being grounded by its true disjuncts 'one loses counterfactual dependence due to grounding overdetermination' is suggestive of the idea that failures of counterfactual dependence, at least with respect to the grounding of disjunctions, always result from grounding overdetermination. 
appears to be a source of failures of counterfactual dependence. We conclude that (ii) is of highly questionable relevance to the matter of the plausibility of (6). Consequently, if we are to make anything much of the observation (i) of the lack of difference-making, this notion will have to be understood in some other way.

We would like to suggest that (i) is best understood in terms of our conception of difference-making. Of course, we cannot interpret (i) as the claim that (6) is an instance of non-difference-making grounding. This would require that (6) is true, which is precisely what is at issue. But it is easy to find a suitably neutral version of this claim. We begin by taking the transitive closure of ground and write $<_{T}$ and $<_{T}$ for the resulting relations; informally, we shall speak of $t$-grounds 20 If grounding is already transitive, but only then, $<_{T}$ and $<_{T}$ will coincide with $<$ and $<$. Now given the assumptions (4) and (5), anyone can agree to the following variant of (6):

$\left(6_{T}\right): b$ is green in spot $s \prec_{T} b$ is largely-red

We may now define a notion of difference-making for $\prec_{T}$ in exact parallel to our previous definition:

(Df. $\left.\prec_{T D}\right)$ : The fact that $P$ is a difference-making partial t-ground of the fact that $Q\left(P \prec_{T D} Q\right)=_{\mathrm{df}}$.

for some scenario $S$ which contains a full t-ground of $Q, S \backslash\{$ the fact that $P$ \} does not contain a full t-ground of $Q$.

$\left(6_{T}\right)$ then turns out to be an example of non-difference-making partial t-grounding. We would like to suggest that this observation nicely captures the intuitive peculiarity of (6) aimed at in Schaffer's remark (i).

Let us turn to remark (iii), that the presence of the green spot in no way helps support the largely-redness of the ball. This again seems a very natural thing to say, and to bring out a further important feature of the case. Nevertheless, it stands in need of explication. In particular, talk of something's helping support a fact is sometimes simply tantamount to an assertion of partial ground. But then (iii) would amount to nothing more than a flat-out denial of (6) rather than give a reason for such a denial. Using our conceptual apparatus, we can give a different, non-question-begging interpretation of (iii). For as we have seen, $\left(6_{T}\right)$ reports a non-difference-making instance of t-grounding: the fact that $b$ is green in spot $s$ is an idle wheel in any full t-ground of the fact that $b$ is largelyred. This, we claim, captures a good sense in which the presence of the green spot in no way helps support the largely-redness of $b$, and it does not beg the question of whether (6) is true or false.

20 More precisely, we let $<_{T}$ be the closure of $<$ under the principle Cut: If $\Gamma<P$ and $P, \Delta<Q$, then $\Gamma, \Delta<Q$. We then let $<_{T}$ be the partial cousin of $<_{T}: P<_{T} Q$ iff $\Gamma<_{T} Q$ for some $\Gamma$ with $P \in \Gamma$. 
The remark (iv) that the green spot is if anything a threat to the largely-redness of the ball $b$ highlights another very important and striking peculiarity of (6). Once more, it cries out for explication. Again using the fact that the presence of the green spot is, if not a ground, still a t-ground, we propose to explicate it in terms of the t-ground-theoretic notions related to difference-making. First, call a partial t-ground of the fact that $Q$ a double-agent iff it would have been a partial t-ground of the fact that $\neg Q$, if $Q$ had not been the case: $: 2122$

(Df. double agent): $P$ is a double agent wrt $Q=_{\mathrm{df}} P \prec_{T} Q$, and

$$
\neg Q \square \rightarrow\left(P \prec_{T} \neg Q\right)
$$

If $b$ had not been largely-red, the fact that it is green in spot $s$ would have been a partial $\mathrm{t}$-ground of the fact that $b$ is not largely-red. So in its role as a t-ground, it pulls equally, as it were, on the side of $b$ 's being largely-red and the side of $b$ 's not being largely-red, simply coming down on whichever side obtains.

It seems, however, that the fact actually pulls more strongly on the side of $b$ 's not being largely-red. We can capture this idea by combining the notion of a double agent with that of difference-making. For if $b$ had not been largely-red, the fact that $b$ is green in spot $s$ would have been a difference-making partial t-ground of the fact that $b$ is not largely-red. In such a case, we shall call a double agent subversive ${ }^{23}$

(Df. subversive): $P$ is a subversive double agent wrt $Q={ }_{\mathrm{df}}$.

$P$ is a double agent wrt $Q, P \nless_{T D} Q$, and $\neg Q \square \leftrightarrow\left(P \prec_{T D} \neg Q\right)$

The observation that the fact that $b$ is green in spot $s$ is a subversive double agent wrt the fact that $b$ is largely-red also seems to nicely capture Schaffer's final remark that the

21 An alternative way to capture the two-faced nature of the relevant kind of ground is by appeal to a suitable non-factive understanding of ground (cf. (Fine, 2012a: 48ff)). Writing $\prec_{T 0}$ for non-factive partial t-ground, we would then count a fact $P$ a double agent wrt $Q$ iff $P<_{T} Q$ and $P \prec_{T 0} \neg Q$. This option promises to yield more satisfactory results in the case of necessarily obtaining groundees $Q$, for which the present proposal counts any partial ground a double agent, assuming the orthodox view that counterfactuals with impossible antecedents are vacuously true. However, since the existence of a clear non-factive understanding of ground may be doubted and since the counterfactual serves our present purposes well enough, we here stick to the counterfactual version.

22 Again, the logic of ground would allow us to systematically produce examples of double agents given suitably independent $P, Q$. For we then have $P \prec(P \wedge Q) \vee(\neg P \wedge Q)$. Now if the groundee had been false, $Q$ would have been false, and we may assume that $P$ would still have been true. But then we would get that $P \prec \neg(\neg P \wedge Q)$, and since $\neg(\neg P \wedge Q) \prec \neg((P \wedge Q) \vee(\neg P \wedge Q))$, by transitivity, $P \prec \neg((P \wedge Q) \vee(\neg P \wedge Q))$, as required for $P$ 's being a double agent.

23 Given $P, Q$, a logical example is given by the true four-way disjunction $R:=(P \wedge Q) \vee(\neg P \wedge Q) \vee$ $(\neg P \wedge \neg Q) \vee Q$. Crucially, if $R$ were false, $P$ would be required to render false the third disjunct of $R$, and thereby would come out a difference-making ground of $\neg R$. 
ball is largely-red despite the green spot, not because of it; or at least, they capture those parts of the remark, which are neutral over the truth of (6). We thus see that our results of the previous section, recast in a form that is neutral over the transitivity of $<$, enables us to give a clear, non-question-begging description of what is so strange about (6) as a putative case of ground. This marks a first serious theoretical payoff of our notion of difference-making, and its strengthening in the notion of a subversive double agent.

In the next section we indicate a further payoff of the conceptual apparatus we have developed by using it to accommodate the counter-intuitive ring of (6) and its kin in a way consistent with the transitivity of ground.

\section{Difference-Making and Explanatory Relevance}

We have observed above that if ground is transitive, then

(6): $b$ is green in spot $s<b$ is largely-red

is a true claim of partial grounding, and one in which the partial ground mentioned fails to be a difference-maker with respect to the fact being grounded. And we have suggested in the previous section that at least a large part of the intuitive reasons for rejecting (6) is captured by the observation that the putative partial ground is a subversive double agent with respect to the fact being grounded.

We still face the question whether (6) is true or false, and relatedly, whether grounding is transitive or not. We know of no decisive considerations with respect to either question, but we incline towards the orthodox view that grounding is indeed transitive and that (6) is accordingly true. Our aim in this section is to use the conceptual apparatus we have put into place thus far to show this view to be defensible.

The general strategy we shall employ may readily be guessed from our introductory remarks at the beginning of this paper. As we have mentioned, it is a familiar idea that a criterion of difference-making for causes may serve to single out a certain elite subclass of the causes of a given event as causal-explanatorily relevant. We suggest that the criterion of difference-making for ( $\mathrm{t}$-)grounds should be accorded an analogous role: it serves not to separate mere t-grounds from real grounds, but rather to separate mere grounds from grounding-explanatorily relevant grounds.

In defence of this suggestion, we will use certain general observations about explanations to argue that one normally cannot adequately explain why a given fact obtains by citing only non-difference-making t-grounds - and especially t-grounds which are subversive double agents with respect to the fact to be explained. This result then allows us to explain the odd ring of (6) without conceding (6) to be false. It can be explained 
as resulting from a general tendency, firstly, to read talk of grounding in terms of 'because', and secondly, to evaluate because-claims as attempts at an explanation, causing us to mistake constraints on good explanations for necessary conditions on grounding.

Following Bromberger (1965) and more recently Schnieder (2015: 183f), we urge that a sharp distinction be made between explaining why something is the case and telling why something is the case. If it is true that $P$ because $Q$, then to successfully tell someone why $P$, it is sufficient to inform the person that $P$ because $Q$. But to successfully explain to the person why $P$, it is also required that the information one provides be sufficient to enable the person to understand why $P$, to solve the epistemic predicament that gave rise to their need for an explanation why $P$.

The act of explaining why something is the case is thus always aimed at resolving some sort of (real or supposed) epistemic predicament. The ability of a ground to figure in a successful grounding explanation is therefore dependent upon its capability to help removing the relevant epistemic predicament. It seems highly plausible that, except perhaps in some exceptional circumstances, only difference-making grounds have this capability. For the special ground-theoretic profiles of non-difference-making grounds and especially subversive double agents make it very hard to see how such grounds could remove any sort of relevant epistemic predicament. The distinctive feature of a non-difference-making ground $f$ is that it does not bring us closer to a full ground of the relevant groundee $g$ : anything we could add to $f$ to obtain a full ground of $g$ already contains such a ground. In light of this, it does not seem as though there could be any sort of puzzlement over how it comes about that the fact $g$ obtains which is even partially resolved by the mention of fact $f$. Assuming $f$ to be a subversive double agent makes the point even more dramatic. For what puzzlement over the question what makes a given fact that $P$ obtain could possibly be removed by the mention of a fact that pulls more strongly on the side of $\neg P$ than it does on the side of $P$ ? In any ordinary context, the information that some ball has a tiny green spot will not remove the epistemic predicament of someone asking why it is almost everywhere red-if anything it will make it worse. We conclude that difference-making, and a fortiori failure to be a subversive double agent, is a plausible minimal requirement on the groundingexplanatory relevance of a ground ${ }^{24}{ }^{25}$

$\overline{24}$ Note that this is our preferred view, but it is not fully mandatory for the arguments in this section. In particular, it would suffice if difference-making was merely a minimal condition for good explanations. The odd ring of the because-claims under discussion could than be blamed on their expressing a particularly bad explanation due to being utterly uninformative and misleading.

25 It might be objected that many grounding claims sound plausible even though they, too, are badly suited to remove any relevant epistemic predicament. For instance, it does not sound implausible—or 
These considerations provide clear grounds for rejecting (or criticizing) grounding explanations given by the assertion of

(7): $b$ is largely-red because $b$ is green in spot $s$

without requiring us to view (7) or (6) as expressing falsehoods. The odd ring of (6) and (7) may accordingly be explained in a way consistent with their truth, and thus with the transitivity of ground, albeit not with the transitivity of grounding-explanation. Note, moreover, that there is precedent for our view in the debate on causal explanation, where the transitivity of causal explanatory relevance is frequently rejected independently of the transitivity of causation 26

\section{Against Schaffer's Contrastive Solution}

Schaffer proposes a different way to salvage the transitivity of grounding against the apparent counter-example (6). His proposal can be seen as involving two parts, which we describe in turn. The first part is an account of grounding as contrastive, coupled with an adjusted notion of transitivity that is applicable to the contrastive conception. On this account, claims of partial grounding take the following canonical form

$$
\left(<_{\text {contrast }}\right) \text { : The fact that } P \text { rather than } P^{*} \text { grounds the fact that } Q \text { rather than } Q^{*} \text {. }
$$
where

$[\mathrm{t}]$ he fact that $[P]$ and the fact that $[Q]$ are required to be obtaining facts, but the fact that $\left[P^{*}\right]$ is required to be a non-obtaining alternative to the fact that $[P]$, and the fact that $\left[Q^{*}\right]$ is required to be a non-obtaining alternative to the fact that $[Q]$. (Schaffer, 2012: 130)

Roughly speaking, what is grounded is never simply a given fact, but that this fact, rather than some given alternative, obtains. And likewise what grounds something is never simply a given fact, but that this fact, rather than some given alternative, obtains. One might therefore say that the relata of ground are not facts but differences - namely, the differences between the relevant obtaining facts and their non-obtaining alternatives.

at least not as implausible as (6) - to say that $P, Q<P \wedge Q$. But would anyone who is puzzled over why $P \wedge Q$ obtains be helped by pointing out that this is because $P$ and $Q$ obtain? In response, we wish to highlight a significant disanalogy. The problem in this last case is one of triviality. Any typical epistemic predicament with respect to $P \wedge Q$ will extend to $P$ and/or $Q$, so the envisaged explanation appeals to facts for which the hearer is also in need of an explanation. The problem in our case of non-difference-making grounding, however, is of a quite different structure. For partially explaining the ball's being largely-red by citing its green spot is bad even if the hearer is not also puzzled over the green spot. Here the problem with the tie appealed to in the explanation is not that it is obvious and thus uninformative, but rather that it has the wrong strength and/or direction, as it were.

26 See Owens (1992: 16ff), Heslow (1981). 
Given this picture of grounding as relating differences, the obvious interpretation of the claim that grounding is transitive is (cf. (Schaffer, 2012, 132)) ${ }^{27}$

( $\mathbf{T}_{\text {contrast }}$ ): If the fact that $P$ rather than $P^{*}$ grounds the fact that $Q$ rather than $Q^{*}$, and the fact that $Q$ rather than $Q^{*}$ grounds the fact that $R$ rather than $R^{*}$, then the fact that $P$ rather than $P^{*}$ grounds the fact that $R$ rather than $R^{*}$

The second part of Schaffer's proposal is the claim that problem cases such as that of the largely-red ball, once recast in explicitly contrastive form, no longer pose a threat to the transitivity of ground as explicated in (T-contrast). For to bring the case into contrastive form, we need to choose suitable alternatives to the three facts involved. And the idea is that however we choose alternatives, either the premises to the transitivitybased inference turn out false, or the conclusion turns out acceptable (cf. (Schaffer, 2012: 136f)).

We may illustrate the difficulties arising for the choice of alternatives as follows. Consider first the fact that our ball $b$ is green in spot $s$. A natural alternative to this fact is that the ball is red in spot $s$. So let us consider as the first relatum in our grounding chain the difference between $b$ being green in spot $s$ and $b$ being red in spot $s$. Next, we need to choose an alternative to the second fact involved, viz. the fact that $b$ has colour distribution $d$. The difference between this fact and the alternative to be chosen must be grounded by the previous difference. The obvious choice is then that $b$ is red all over. So our first contrastive grounding claim reads:

(4*): The fact that $b$ is green rather than red in spot $s$ grounds the fact that $b$ has colour distribution $d$ rather than being red all over.

Finally, we need to choose an alternative to the third fact involved, viz. that $b$ is largelyred. But now we are in trouble, for both $b$ having colour distribution $d$ and $b$ being red all over are ways for $b$ to be largely-red. So that $b$ is one rather than the other cannot plausibly be taken to ground that $b$ is largely-red rather than something else.

$\left(5^{*}\right)$ : The fact that $b$ has colour distribution $d$ rather than being red all over grounds the fact that $b$ is largely-red rather than ???

So if we choose our first two alternatives in the way we did, we do not obtain a threat to transitivity.

Unfortunately, there are other possible choices of alternatives which seem much more troublesome. Call $d^{\prime}$ a possible colour distribution for $b$ which is like $d$ except in that it has $b$ red in spot $s$, but green in half of its total surface area. Then:

$\overline{27}$ Note that this picture also yields a very strong and very literal connection between grounding and difference-making, in that it portrays ground simply as the making of one difference by others. 
(5'): The fact that $b$ has colour distribution $d$ rather than $d^{\prime}$ grounds the fact that $b$ is largely-red rather than half green.

Leaving the first choice of alternative as it was, we obtain the following contrastive variant on (4):

(4'): The fact that $b$ is green rather than red in spot $s$ grounds the fact that $b$ has colour distribution $d$ rather than $d^{\prime}$.

This is plausible. Consider the overall difference between the colour distributions $d$ and $d^{\prime}$. Part of that difference is that $d$ has $b$ green in spot $s$, whereas $d^{\prime}$ has $b$ red in spot $s$. And that part of the overall difference is fully accounted for by the fact that $b$ is green rather than red in spot $s$. Therefore the fact that $b$ is green rather than red partially grounds the fact that $b$ has colour distribution $d$ rather than $d^{\prime}$.

We now have a pair of contrastive grounding claims, namely $\left(4^{\prime}\right)$ and $\left(5^{\prime}\right)$, to which the contrastive transitivity principle $\left(\mathrm{T}_{\text {contrast }}\right)$ applies. We then obtain

$\left(6^{\prime}\right)$ : The fact that $b$ is green rather than red in spot $s$ grounds the fact that $b$ is largely-red rather than half green.

But if anything, this sounds even worse than (6). So moving to a contrastive conception of grounding does nothing to alleviate the challenge to transitivity from cases like the largely-red ball.

Of course, Schaffer could try to devise some criterion that prohibits the choice of contrasts we have used to obtain $\left(6^{\prime}\right)$. It is, however, far from obvious how this might be done. Indeed, Schaffer himself readily admits that he is in no possession of objective criteria for the selection of contrasts, and even exhibits a slight pessimism as to whether such criteria may be found at all ${ }^{28}$ Meanwhile, given that Schaffer himself favours a view according to which grounding is a relation that backs explanation, our way to explain the odd ring of (6) and (7) while retaining the transitivity of ground should certainly not be inherently objectionable to him.

\section{Going Further: Proportionality and Cohesion}

There are various directions in which the present account of difference-making for grounding and its relation to grounding explanation may be further developed. We'd like to conclude this paper by tentatively exploring one of them.

Note that the notion of difference-making we have proposed only allows for two choices with respect to the partial grounds of a given fact: either a given partial ground makes a difference to the obtaining of the fact, or it does not. Yet, with the notions of being a double-agent and a subversive double-agent, we are in the position to also 28 Cf. Schaffer $(2016,68)$. 
make rough qualitative distinctions within the realm of non-difference-making partial grounds. Double-agents and subversive double-agents can be seen as two increasingly 'severe' forms of non-difference-making, which, as we have suggested, may in turn correspond to increasingly severe forms of explanatory irrelevance. In order to account for these forms of non-difference-making, we have used counterfactuals (though there might be a more elegant solution using a non-factive understanding of grounding). A natural question is whether an appeal to counterfactuals would also allow us to make helpful qualitative distinctions within the realm of difference-making grounds that in turn may afford useful distinctions with respect to the explanatory relevance of difference-making partial grounds.

A very straightforward idea that suggests itself is to combine our notion of differencemaking with a certain form of counterfactual dependence. The idea is best illustrated by an example. Consider the following case. Call an object signal-coloured iff it is either red, yellow, or green. Suppose that some street-sign $s$ has some fully determinate shade of red, $C$. Now, that $s$ has $C$ fully grounds $s$ 's having the determinable colour red. The latter, in turn is a full ground of the fact that $s$ is signal-coloured. Since every full ground is a difference-maker, it follows by transitivity that

(8): $s$ has $C \prec_{T D} s$ is signal-coloured, and

(9): $s$ is red $\prec_{T D} s$ is signal-coloured.

Arguably, (8) and (9) differ in an important respect. Roughly speaking, even though the fact that $s$ is $C$ makes a difference to $s$ 's being signal-coloured in our sense, it does not seem to be the most relevant bit of information in that respect. For, if $s$ would not have this particular shade of red, but a slightly different one, it would still be signal-coloured (a bit more technical: in the closest non- $C$ worlds, $s$ will still have some determinate shade of red). On the other hand, if $s$ would not have been red, but some other colour, it might not have been signal-coloured (a bit more technically: among the closest nonred worlds, there are some worlds in which $s$ is beige, blue, etc.). We can capture this difference between (8) and (9) by calling the former a case of weak difference-making and the latter a case of strong difference-making. Strong difference-making (in symbols $\prec_{T D}^{!}$) can be defined as follows:

(Df. Strong): $P \prec_{T D}^{!} Q=_{\text {df. }} P \prec_{T D} Q$ and $\neg(\neg P \square \leftrightarrow Q)$.

The distinction between weak and strong difference-makers can naturally be related to the notion of explanatory relevance. At least in many cases, weak difference-makers seem to be too specific to satisfactorily explain why a given fact obtains. That the sign $s$ is signal-coloured is not explained by the fact that it has this particular shade of red, but rather by the fact that it has some shade of red. Since any other shade of red among the 
array of different specific shades of red incompatible with $C$ would have done as well, information about the specific shade seems explanatorily irrelevant with respect to the fact that $s$ is signal-coloured. ${ }^{29}$

Notably, our case instantiates a pattern that is widely discussed in the debate on causal difference-making and causal explanation ${ }^{30}$ In particular, on Strevens' account of causal explanatory relevance, it is a requirement for a causal influence on a given event to be explanatorily relevant that it is characterized in a sufficiently unspecific way. To pick an illustrative example from Strevens (2008, 96), suppose that a given cannonball is shot at a window and shatters it. The specific weight of the ball, say $10.208 \mathrm{~kg}$, is among the causal influences that bring about the window's shattering. However, thus Strevens', the ball's specific weight is explanatorily irrelevant with respect to the window's shattering. All else being equal, any ball with a weight of more than $5 \mathrm{~kg}$ would have broken the window. Hence, thus his suggestion, what makes a difference that is explanatorily relevant to the window's shattering is not the ball's having some determinate mass, but rather its having the determinable property of weighing more than $5 \mathrm{~kg} .^{31}$

Just as in Strevens' causal case, the strong difference-maker in our case seems to have an explanatory advantage over the weak one because it instantiates a more general pattern and affords, in that sense, a greater unification than weak difference-makers. With respect to the question of why $s$ is signal-coloured, the answer that $s$ is red is invariant over a variety of mutually incompatible determinate shades of red.

It may be, however, that the aforementioned answer is not the most general answer one can give. The reason is that the class of strong difference-makers may not be exhausted by s's being red; $s$ 's being either red or green may also count as a strong difference-maker for $s$ 's being signal-coloured. Clearly, the closest worlds in which $s$ is neither red nor green include worlds in which $s$ is not signal-coloured. So assuming that the fact that $s$ is either red or green is a ground of the fact that $s$ is signal-coloured,

29 We can, at this point, stay agnostic with respect to the issue whether being a strong difference-maker merely determines pragmatic acceptability of certain because-claims, or whether it captures an objective criterion of explanatory relevance.

30 See e.g. Strevens (2008: §3.5), Sartorio (2005: 75), and cf. Yablo (1992; §4).

31 Of course the specific weight $i$ s causally responsible for specific features of the window's breaking (for how it breaks exactly) in a way the determinable weight is not. Strevens assumes, however, a scenario wherein the explanandum in question is not the window's breaking-in-a-highly-specific-way, but rather its simply breaking. This seems plausible: in any ordinary sense of 'explanation', there is a wide array of explanations of the occurrence of some given event, where highly specific details of how the event occurred are simply beside the question. Compare on this also Schaffer (2012, 135). 
it is also strong difference-maker. ${ }^{32}$ It seems, however, that an explanation of $s$ 's being signal-coloured in terms of its being either red or green is also unsatisfactory, or at least less satisfactory than the one in terms of $s$ 's being red. Hence, being a strong difference-maker may only be a necessary condition for being explanatorily relevant.

So one may wish to hold that while weak difference-makers, like s's specific colour, may be too informative, some strong difference-makers, like the disjunctive property of having one of the two colours red and green, seem too uninformative ${ }^{33}$ The natural idea is then to say that for a ground to be explanatorily relevant, it must be unspecific enough to be a strong difference-maker, but among the strong difference-makers, it must be maximally informative and specific. At least in many cases, it seems to us, an appropriate standard for comparative informativeness is given by saying that the fact that $P$ is more informative than the fact that $Q$ iff $P<Q$. Our considerations then suggest that to be explanatorily relevant, a partial ground of a fact must be a cohesive difference-making ground of the fact, where this is understood thus:

(Df. Cohesive): $P$ is a cohesive difference-making ground of $Q={ }_{\mathrm{df}} P<_{T D} Q$, and there is no $P^{\prime}$ such that $P^{\prime}$ is a strong difference-maker wrt $Q$ and $P^{\prime}<P$.

A cohesive difference-making ground of a given fact is a maximally informative strong difference-maker with respect to the fact. Specifying cohesive difference-makers seems to be a plausible desideratum for good grounding-explanations that mirrors comparable desiderata that have been proposed for causal explanations. ${ }^{34}$ Of course, in order to substantiate this proposal, it would be desirable to corroborate it with more data than the example we have considered, and also to sketch its relation to other, extant theories of explanatory relevance. To explore these issues must, however, be deferred to future research.

32 Whether the ground-theoretic assumption holds depends on some subtle details of one's theory of ground and of how the property of being signal-coloured is conceived. For the sake of argument, however, we grant the ground-theoretic assumption to our opponent.

33 See Strevens (2008. 101ff). We adopt the terminology of a cohesive difference-maker from him.

34 Note that in the debate on the metaphysics of causation, notions of difference-making that follow an idea analogous to the one we have been developing in this section have been suggested as necessary conditions for being a causal influence (often under the label 'proportionality'); cf. e.g. Yablo (1992, 273ff), and Sartorio (2005). For reasons outlined earlier (sec. 5), we prefer to view cohesive difference-making as a condition (or a good-making feature) of explanations, rather than as a condition for the obtaining of the respective explanation-backing relation. A view congenial to ours is that of Weslake (2013; §6) who defends the idea that proportionality is a dimension of explanatory value. 


\section{Conclusion}

Sometimes, the grounds of a given fact give rise to good grounding-explanations of the fact, but sometimes it appears that they do not. Likewise, sometimes the causes of a given event give rise to good causal explanations of the event's occurrence, and sometimes it appears that they do not. To delineate the class of explanatorily relevant causes of an event, some authors have appealed to a notion of difference-making, and proposed explications of that notion designed for the application to causes. In this paper, we have explored the potential for a parallel move in the case of grounding. We have firstly, described a notion of difference-making that applies to the partial grounds of a fact, and we have made a strong case that there are grounds on both sides of the division induced by the notion. We have also introduced a number of significant subdivisions within the realms of both difference-making and non-difference-making partial grounds. We have then made a preliminary case that these divisions among the grounds of a fact correspond to qualitative differences between candidate grounding-explanations of the fact. In addition, we have applied our conceptual apparatus to the analysis of certain putative counter-examples to the transitivity of grounding, and we have argued that these may naturally be accommodated within our framework as mere failures of transitivity for good grounding explanations. This result is significant especially because, as we have also shown, Schaffer's alternative strategy to accommodate the counter-examples within a contrastive account of grounding fails to remove the threat to transitivity.

A number of questions remain open. The contention that our notions of differencemaking relate in the way suggested to explanatory relevance has to be checked against a wide range of data. Undoubtedly, many other natural subdivisions among the difference-makers and non-difference-makers of the grounds of a fact that are similar to ours may be made, and their relations to each other and to explanation examined. And of course, the theory of the various kinds of grounds we have distinguished, if they are found fruitful, still needs to be developed. What we hope to have shown, in addition to the more local results stated above, is that these open questions constitute worthwhile avenues for further research.

\section{Appendix A. Logical Cases of Non-Difference-Making}

We have claimed above that plausible assumptions concerning the logic of ground allow us to identify a systematic way of producing instances of non-difference-making ground given a pair of suitably independent facts to start with. This appendix develops our above, rough and informal argument for this claim in more detail. 
The required logical assumptions concern the pure and the propositional logic of ground. Unfortunately, there is currently no standard, fully worked out propositional logic of ground. Rather, what we have is: (I) a system of natural inference rules that are plausible relative to a very fine-grained conception of ground, proposed by Kit Fine, but with as yet no adequate semantics, and acknowledged as incomplete with respect to any plausible understanding of ground (cf. (Fine, 2012a: 67)); (II) a natural truthmakersemantics, also proposed in Fine (2012a), which yields a logic adequate to a much more coarse-grained conception of ground; (III) a logical system proposed by Correia (2010), addressing again a coarse-grained conception of ground, proven sound and complete for a corresponding algebraic semantics ${ }^{35}$ An additional difficulty is that the languages of these logical systems are expressively too weak to state anything like the quantified claim that for every $\Gamma$, if $\Gamma, P<Q$, then $\Gamma^{0}<Q$ for some $\Gamma^{0} \subseteq \Gamma$ with $P \notin \Gamma^{0}$.

Nevertheless, we believe a useful case for our claim can be made. To this end, we shall do two things. Firstly, we discuss a sharpening of our above informal argument in the context of the truthmaker-semantics for ground. We suggest that in this context, it is best to work with a slightly more refined conception of what it is for $\Gamma$ to contain a full ground of some fact than the simple set-theoretic conception employed above. It can then be shown that for suitably independent, true $P$ and $Q$, the informal argument given above goes through. It would take a lot of work to reconstruct the argument within the logic of (III), so we choose not to do so here. However, it is known that the logic obtained on the semantics in (II) is very close to that in (III) (cf. Fine (ms: 11)), and it is clear that a version of our argument can be given for Correia's system, too. Secondly, we discuss our informal argument in the context of the system (I). We show that given an additional rule that turns out valid on both of the only two semantics known to us that validate the others of Fine's rules, our argument goes through given very modest assumptions of independence of $P$ and $Q$. Without the additional rule, a move parallel to that made before of refining the relevant notion of containment will secure our result.

A.1. The Coarse-Grained Framework. In his truthmaker-semantics for ground, Fine associates with each sentence $A$ a set of states that verify $A$ and a set of states that falsify $A$. States are here thought of as obtaining. Since no sentence is both true and false and no sentence is neither, exactly one of the two sets of states associated with a given sentence is empty $\sqrt{66}$ For our purposes, it is easiest to reason directly about the

35 The fineness of grain of a conception of ground is a matter of the conditions under which sentences are substitutable salva veritate in the scope of a grounding-operator. More coarse-grained conceptions allow more substitutions, more fine-grained conceptions less.

36 Fine in 2012a) actually speaks of facts rather than states. Since we have used 'fact' for the relata of the grounding relation facts, to avoid terminological confusion, we use 'state' instead. (Note that in 
sets of states and forget about the sentences to which they are assigned. We may then think of a non-empty set of states as a true proposition which is verified by exactly its members. Following Fine, we assume that (i) whenever there are states $s, t, u, \ldots$, there is also their fusion $\bigsqcup\{s, t, u, \ldots\}=s \sqcup t \sqcup u \sqcup \ldots .{ }^{37}$ and that (ii) any proposition $P$ is closed under non-empty fusions, so that $\sqcup P^{\prime} \in P$ whenever $\emptyset \subset P^{\prime} \subseteq P$.

The full and partial notions of (strict) ground are defined by Fine in terms of prior notions of full and partial weak ground. The definitions are as follows:

$$
\begin{aligned}
& \text { (Df. } \leq \text { ): } P_{1}, P_{2}, \ldots \leq Q==_{\text {df. }} s_{1} \sqcup s_{2} \sqcup \ldots \in Q \text { whenever } s_{1} \in P_{1}, s_{1} \in P_{2}, \ldots \\
& \text { (Df. } \leq \text { ): } P \leq Q={ }_{\text {df. }} \Gamma, P \leq Q \text { for some set of propositions } \Gamma \\
& \text { (Df. }<\text { ): } P_{1}, P_{2}, \ldots<Q==_{\text {df. }} P_{1}, P_{2}, \ldots \leq Q \text { and } Q \npreceq P_{i} \text { for all } i \\
& \text { (Df. }<\text { ): } P<Q=_{\text {df. }} P \leq Q \text { and } Q \not P
\end{aligned}
$$

Now say that a state $s$ is part of a state $t$ iff for some state $s^{\prime}, t=s \sqcup s^{\prime}$, and say that two states $s$ and $t$ overlap iff they share some (non-nul[ $\left.{ }^{38}\right)$ part.

The conjunction $P \wedge Q$ of two propositions is the set $\{s \sqcup t: s \in P$ and $t \in Q\}$, and the disjunction $P \vee Q$ is the set $P \cup Q \cup(P \wedge Q)$. Now consider any truths $P, Q$ which are independent in the sense that $\bigsqcup P$ and $\bigsqcup Q$ do not overlap. We may now show that

$$
\text { (A): } P<Q \vee(P \wedge Q)
$$

Firstly, note that $P, Q<Q \vee(P \wedge Q)$. For suppose $s \in P$ and $t \in Q$. Then $s \sqcup t \in P \wedge Q$, and hence $s \sqcup t \in Q \vee(P \wedge Q)$. So $P, Q \leq Q \vee(P \wedge Q)$, and hence $P \leq Q \vee(P \wedge Q)$. But now consider $\bigsqcup(Q \vee(P \wedge Q))=\sqcup P \sqcup \sqcup Q$. Since $\sqcup P$ and $\sqcup Q$ do not overlap, $\sqcup P \sqcup \sqcup Q$ is not part of $\bigsqcup P$. But it is easy to verify that $R \leq P$ only if $\bigsqcup R$ is part of $\sqcup P$, so it follows that $Q \vee(P \wedge Q) \npreceq P$. Hence $P<Q \vee(P \wedge Q)$.

Now suppose that some scenario $S$ which includes the fact that $P$ contains a scenario which is a strict full ground of $Q \vee(P \wedge Q)$. To show that $P$ is not a difference-maker, we need to show that $S \backslash\{$ the fact that $P$ \} still contains a strict full ground of $P$. If this fails for any scenario, it fails for a scenario which is a strict full ground of $Q \vee(P \wedge Q)$, so we may restrict attention to such scenarios. Suppose, therefore, that

$$
\text { (H): } \Gamma, P<Q \vee(P \wedge Q)
$$

other versions of his truthmaker semantics, Fine also appeals to states, and then typically allows nonactual and indeed often impossible states alongside the actual, obtaining ones. So we should perhaps emphasize again that we here restrict 'state' to actual, obtaining states.)

37 Fusion is assumed to be associative, so that $\sqcup P_{0} \sqcup \sqcup P_{1} \sqcup \ldots=\sqcup\left(P_{0} \cup P_{1} \cup \ldots\right)$. Use of this fact will often be tacit in what follows.

38 Since fusion is defined for the empty set of states, we always have a minimal state which is part of every state, called the nullstate. The relation of overlap must therefore be taken to require sharing of non-null parts, otherwise it trivializes. 
It follows that every verifier of $\wedge \Gamma \wedge P$ is a verifier of $Q \vee(P \wedge Q)$. Since any such verifier has a part that verifies $P$, by the assumption of independence, no such verifier is a verifier of $Q$, and hence it must be a verifier of $P \wedge Q$. But since no verifier of $P$ is part of a verifier of $Q$, it follows that every verifier of $\bigwedge(\Gamma \backslash\{P\})$ must have a part that verifies $Q$.

But does it follow that $\Gamma \backslash\{P\}$ contains a strict full ground of $Q \vee(P \wedge Q)$ ? If a scenario contains another just in case the latter is a subset of the former, it does not ${ }^{39}$ In a rough approximation, the problem is that although some subset of $\Gamma \backslash\{P\}$ must be strong enough to establish $Q$, it may be that every such subset is, as it were, strictly between $Q$ and $P \wedge Q$, and thereby fail to ground $Q \vee(P \wedge Q) .40$

However, intuitively, what this shows is not that $P$ may after all be a difference-maker with respect to $Q \vee(P \wedge Q)$, but that the set-theoretic interpretation of the containment of one scenario in another is unsatisfactory. For in the intuitively relevant sense, since every verifier of $\bigwedge(\Gamma \backslash\{P\})$ contains a verifier of $Q, \Gamma \backslash\{P\}$ contains $\{Q\}$, and thereby a strict full ground of $Q \vee(P \wedge Q)$. So what we should do is refine the conception of containment appealed to in the definition of difference-making. We shall therefore say that a scenario $\Gamma$ contains a scenario $\Gamma^{0}$ just in case every verifier of $\wedge \Gamma$ has a part that verifies $\wedge \Gamma^{0}$. Then since $\Gamma \backslash\{P\}$ contains $\{Q\}$ whenever $\Gamma, P<Q \vee(P \wedge Q), P$ comes out a non-difference-making partial ground of $Q \vee(P \wedge Q)$.

A.2. The Fine-Grained Framework. We use the following rules proposed by Fine:

$\operatorname{Sub}(</<)$ : From $\Gamma, A<B$ infer $A<B$

$\operatorname{Sub}(<\mid \leq)$ : From $\Gamma<B$ infer $\Gamma \leq B$

$\operatorname{Sub}(\leq / \leq)$ : From $\Gamma, A \leq B$ infer $A \leq B$

$\operatorname{Sub}(</ \leq)$ : From $A<B$ infer $A \leq B$

Trans $(</<)$ : From $A<B$ and $B<C$ infer $A<C$

39 Here is a counter-example. Suppose that $P=\{s\}, Q=\{t\}$, where $t$ and $s$ do not overlap. Then $Q \vee(P \wedge Q)=\{t, s \sqcup t\}$. Now suppose $R=\{u\}$ with $u$ strictly between $t$ and $s \sqcup t$. Note that $s \sqcup u=s \sqcup t$. Now consider $\Gamma=\{R\}$. Then $\Gamma, P \leq Q \vee(P \wedge Q)$. But as before, $Q \vee(P \wedge Q) \not \subset$, and likewise $Q \vee(P \wedge Q) \npreceq R$, since $u$ is a proper part of $s \sqcup t$. So $\Gamma, P<Q \vee(P \wedge Q)$. But $R \nsubseteq Q \vee(P \wedge Q)$, so $R \not \leq Q \vee(P \wedge Q)$, and a fortiori, $R \nless Q \vee(P \wedge Q)$.

40 Note that if we were to assume that every proposition $P$ isconvex in the sense that $u \in P$ whenever $s, t \in P, s$ is part of $u$, and $u$ is part of $t$, this case cannot obtain. This is significant given that according to Fine (ms: 11), grounding defined as above on convex propositions coincides with ground as per the logic of Correia (2010). So it would appear that with respect to Correia's logic, the argument for $P$ being a non-difference-making partial ground of $Q \vee(P \wedge Q)$ indeed goes through in its original form. For criticism of the convexity constraint and the corresponding principle in Correia's logic, see Krämer and Roski (2015) and Correia (2015). 
$\operatorname{Trans}(\leq /<)$ : From $\Gamma \leq A$ and $A<B$, infer $\Gamma<B$

Trans $(</ \leq)$ : From $A<B$ and $B \leq C$ infer $A<C$

$\operatorname{Irr}(<)$ : From $A<A$, infer $\perp$

$\operatorname{Rev} \operatorname{Sub}(\leq /<)$ : From $A_{1}, A_{2}, \ldots \leq B$ and $A_{1}<B, A_{2}<B, \ldots$, infer $A_{1}, A_{2}, \ldots<B$

$(\wedge \mathbf{I})$ : From $A$ and $B$, infer $A, B<A \wedge B$

$(\vee \mathbf{I})$ : From $A$, infer $A<A \vee B$ or $B<A \vee B$

$(\vee \mathbf{E})$ : From $\Gamma<A \vee B$, infer that either $\Gamma \leq A$, or $\Gamma \leq B$, or for some $\Gamma_{A}, \Gamma_{B}$ with $\Gamma=\Gamma_{A} \cup \Gamma_{B}: \Gamma_{A} \leq A$ and $\Gamma_{B} \leq B$

$(\wedge \mathbf{E})$ : From $\Gamma<A \wedge B$ infer that for some $\Gamma_{A}, \Gamma_{B}$ with $\Gamma=\Gamma_{A} \cup \Gamma_{B}: \Gamma_{A} \leq A$ and $\Gamma_{B} \leq B$

We make the following assumptions.

(A1): $P$

(A2): $Q$

(A3): $P \npreceq Q$

Using the rules $(\wedge \mathrm{I}),(\vee \mathrm{I})$, and $\operatorname{Trans}(<)$, we obtain

(1): $P<Q \vee(P \wedge Q)$

Now suppose

(S): $\Gamma, P<Q \vee(P \wedge Q)$

By $(\mathrm{VE})$, we may infer from $(\mathrm{S})$ that one of the following three claims holds:

(i): $\Gamma, P \leq Q$

(ii): $\Gamma, P \leq P \wedge Q$

(iii): For some $\Delta_{1}, \Delta_{2}$ with $\Delta=\Delta_{1} \cup \Delta_{2}: \Delta_{1} \leq Q$ and $\Delta_{2} \leq P \wedge Q$

But from (i), it follows by $\operatorname{Sub}(\leq / \leq)$ that $P \leq Q$, contrary to our assumption.

Now suppose (iii), and let $\Delta_{1} \leq Q$. Note that $P \notin \Delta_{1}$, for otherwise again $P \leq Q$, contrary to our assumption. So $\Delta_{1}$ is a subset of $\Gamma \cup\{P\}$, not including $P$, with $\Delta_{1} \leq Q$. Using that $Q<Q \vee(P \wedge Q)$ as well as Trans $(\leq /<)$, we obtain that $\Delta_{1}<Q \vee(P \wedge Q)$. So $\Delta_{1}$ is the required witness for the claim that $P$ is a non-difference-making partial ground of $Q \vee(P \wedge Q)$ for the case that (iii) holds.

Suppose finally that (ii) holds. Then by $\operatorname{Sub}(\leq / \leq)$, we have $R \leq P \wedge Q$ for all $R \in \Gamma, P$. Since any relationship of weak partial ground is either strict or mutual, it follows by Rev $\operatorname{Sub}(\leq /<)$ that one of the following two conditions holds:

(a): $\Gamma, P<P \wedge Q$

(b): $P \wedge Q \leq R$ for some $R \in \Gamma, P$

If (a), then by $(\wedge \mathrm{E})$ we obtain that for some subset $\Delta_{1}$ of $\Gamma, P, \Delta_{1} \leq Q$. Note that $P \notin \Delta_{1}$ for otherwise $P \leq Q$, contrary to our assumption. So $\Delta_{1}$ is a subset of $\Gamma \cup\{P\}$, not 
including $P$, with $\Delta_{1} \leq Q$, and thereby as before, $\Delta_{1}<Q \vee\left(P \wedge Q\right.$. So $\Delta_{1}$ is the required witness for the claim that $P$ is a non-difference-making partial ground of $Q \vee(P \wedge Q)$ for the case that (ii.a) holds.

If (b) holds, then we have for some $R \in \Gamma \cup\{P\}$ both $P \wedge Q \leq R$ and $R \leq P \wedge Q$. It is tempting to infer from this that $P \wedge Q$ and $R$ are mutual weak full grounds, as per the rule:

$\operatorname{Rev} \operatorname{Sub}(\leq / \leq)$ : From $A \leq B$ and $B \leq A$, infer $A \leq B$

And on the only proposals for a semantics for a logic of ground incorporating Fine's rules - in as yet unpublished work by Krämer $(\mathrm{ms})$ and Correia $(\mathrm{ms})$ - this inference indeed comes out valid. But then $R \leq P \wedge Q$, and since $P \wedge Q<Q \vee(P \wedge Q)$ we obtain $R<Q \vee(P \wedge Q)$. Now it can be shown that $R \neq P$. For otherwise we obtain $P \leq P \wedge Q$. But $P \wedge Q \npreceq P$, for otherwise we obtain by Trans $(</ \leq)$ that $P<P$, and thus $\perp$ by $\operatorname{Irr}(<)$. It follows by $\operatorname{Rev} \operatorname{Sub}(\leq /<)$ that $P<P \wedge Q$. But then by the elimination rule $(\wedge \mathrm{E})$, $P \leq Q$ and hence $P \leq Q$, contrary to our assumption. So $P \notin\{R\}$, and hence $\{R\}$ is our required witness for the claim that $P$ is a non-difference-making partial ground of $Q \vee(P \wedge Q)$ for the case that (iii) holds.

Now it may be that there could also be a plausible semantics for the kind of logic of ground Fine proposes which delivers natural counter-models to Rev $\operatorname{Sub}(\leq / \leq)$. In that case $R$ would again, roughly speaking, have to lie between $Q$ and $P \wedge Q$ in strength. But then similar means to those proposed above would give a natural way to salvage our case by refining the conception of containment to fit the relevant sense of 'between'.

\section{REFERENCES}

Audi, P. (2012a). A Clarification and Defense of the Notion of Grounding. In F. Correia and B. Schnieder (Eds.), Metaphysical Grounding, pp. 101-121. Cambridge, UK: Cambridge University Press.

Audi, P. (2012b). Grounding: Toward a Theory of the in-virtue-of Relation. The Journal of Philosophy 109(12), 685-711.

Bromberger, S. (1965). An Approach to Explanation. In R. J. Butler (Ed.), Analytical Philosophy, Volume 2, pp. 72-105.

Correia, F. (2010). Grounding and Truth-Functions. Logique et Analyse 53(211), 251279.

Correia, F. (2015). On the Logic of Factual Equivalence. The Review of Symbolic Logic FirstView.

Correia, F. (ms). An Impure Logic of Representational Grounding. Unpublished manuscript. 
Correia, F. and B. Schnieder (2012). Grounding: An Opinionated Introduction. In F. Correia and B. Schnieder (Eds.), Metaphysical Grounding: Understanding the Structure of Reality, pp. 1-36. Cambridge: Cambridge University Press.

Fine, K. (2012a). Guide to Ground. In B. Schnieder and F. Correia (Eds.), Metaphysical Grounding, pp. 37-80. Cambridge: Cambridge University Press.

Fine, K. (2012b). The Pure Logic of Ground. The Review of Symbolic Logic 5(1), 1-25.

Fine, K. (ms). A Theory of Truth-Conditional Content II. Unpublished manuscript.

Heslow, G. (1981). The Transitivity of Causation. Analysis 41(3), 130-133.

Krämer, S. (2016). A Hyperintensional Criterion of Irrelevance. Synthese, 1-14.

Krämer, S. (ms). Towards a Theory of Ground-Theoretic Content. Unpublished manuscript.

Krämer, S. and S. Roski (2015). A Note on the Logic of Worldly Ground. Thought: A Journal of Philosophy 4(1), 59-68.

Lewis, D. (1973). Counterfactuals. Oxford: Blackwell.

Lewis, D. (1986a). Causal Explanation. In D. Lewis (Ed.), Philosophical Papers, Volume II, pp. 214-240. Oxford and New York: Oxford University Press.

Lewis, D. (1986b). Causation. In D. Lewis (Ed.), Philosophical Papers, Volume II, pp. 159-213. Oxford and New York: Oxford University Press.

List, C. and P. Menzies (2009). Nonreductive Physicalism and the Limits of the Exclusion Principle. Journal of Philosophy, 475-502.

Litland, J. E. (2013). On Some Counterexamples to the Transitivity of Grounding. Essays in Philosophy 14(1), 19-32.

Owens, D. (1992). Causes and Coincidences. Cambridge, UK: Cambridge University Press.

Ruben, D.-H. (2012). Explaining Explanation (Updated and Extended Second Edition). Boulder: Paradigm Publishers.

Sartorio, C. (2005). Causes as Difference-Makers. Philosophical Studies 123, 71-96.

Schaffer, J. (2012). Grounding, Transitivity, and Contrastivity. In B. Schnieder and F. Correia (Eds.), Metaphysical Grounding, pp. 122-138. Cambridge: Cambridge University Press.

Schaffer, J. (2016). Grounding in the Image of Causation. Philosophical Studies 173(1), 49-100.

Schnieder, B. (2010). A Puzzle about 'Because'. Logique et Analyse 53(211), 317-343.

Schnieder, B. (2015). The Asymmetry of 'Because'. Grazer Philosophische Studien 91, 175-208.

Strevens, M. (2008). Depth. Cambridge Mass.: Haward University Press. 
Trogdon, K. (2013). An Introduction to Grounding. In M. Hoeltje, B. Schnieder, and A. Steinberg (Eds.), Varieties of Dependence, pp. 97-122. Munich: Philosophia.

Weslake, B. (2013). Proportionality, Contrast, and Explanation. Australasian Journal of Philosophy 91(4), 785-797.

Woodward, J. (2003). Making Things Happen. Oxford and New York: Oxford University Press.

Yablo, S. (1992). Mental Causation. The Philosophical Review 101(2), 245-280. 